\title{
Multijoint Muscle Regulation Mechanisms Examined by Measured Human Arm Stiffness and EMG Signals
}

\author{
RIEKO OSU ${ }^{1}$ AND HIROAKI GOMI ${ }^{2,3}$ \\ ${ }^{1}$ Kawato Dynamic Brain Project, ERATO, Japan Science and Technology Corporation, Kyoto, 619-0288; ${ }^{2}$ NTT \\ Communication Science Labs, Nippon Telegraph and Telephone Corporation, Kanagawa, 243-0198; \\ and ${ }^{3}$ CREST, Japan Science and Technology Corporation, Ibaraki, 305-8568, Japan
}

\begin{abstract}
Osu, Rieko and Hiroaki Gomi. Multijoint muscle regulation mechanisms examined by measured human arm stiffness and EMG signals. $J$. Neurophysiol. 81: 1458-1468, 1999. Stiffness properties of the musculoskeletal system can be controlled by regulating muscle activation and neural feedback gain. To understand the regulation of multijoint stiffness, we examined the relationship between human arm joint stiffness and muscle activation during static force control in the horizontal plane by means of surface electromyographic (EMG) studies. Subjects were asked to produce a specified force in a specified direction without cocontraction or they were asked to keep different cocontractions while producing or not producing an external force. The stiffness components of shoulder, elbow, and their cross-term and the EMG of six related muscles were measured during the tasks. Assuming that the EMG reflects the corresponding muscle stiffness, the joint stiffness was predicted from the EMG by using a two-link six-muscle arm model and a constrained least-squareerror regression method. Using the parameters estimated in this regression, single-joint stiffness (diagonal terms of the joint-stiffness matrix) was decomposed successfully into biarticular and monoarticular muscle components. Although biarticular muscles act on both shoulder and elbow, they were found to covary strongly with elbow monoarticular muscles. The preferred force directions of biarticular muscles were biased to the directions of elbow monoarticular muscles. Namely, the elbow joint is regulated by the simultaneous activation of monoarticular and biarticular muscles, whereas the shoulder joint is regulated dominantly by monoarticular muscles. These results suggest that biarticular muscles are innervated mainly to control the elbow joint during static force-regulation tasks. In addition, muscle regulation mechanisms for static force control tasks were found to be quite different from those during movements previously reported. The elbow single-joint stiffness was always higher than cross-joint stiffness (off-diagonal terms of the matrix) in static tasks while elbow single-joint stiffness is reported to be sometimes as small as cross-joint stiffness during movement. That is, during movements, the elbow monoarticular muscles were occasionally not activated when biarticular muscles were activated. In static tasks, however, monoarticular muscle components in single-joint stiffness were increased considerably whenever biarticular muscle components in single- and cross-joint stiffness increased. These observations suggest that biarticular muscles are not simply coupled with the innervation of elbow monoarticular muscles but also are regulated independently according to the required task. During static force-regulation tasks, covariation between biarticular and elbow monoarticular muscles may be required to increase stability and/or controllability or to distribute effort among the appropriate muscles.
\end{abstract}

\section{N T R O D U C T I O N}

The human musculo-skeletal system has springlike properties that enable posture stabilization and interaction with the

\footnotetext{
The costs of publication of this article were defrayed in part by the payment of page charges. The article must therefore be hereby marked "advertisement" in accordance with 18 U.S.C. Section 1734 solely to indicate this fact.
}

environment. Stiffness properties are determined by both the muscle's inherent springlike properties and neural feedback systems, and the stiffness can be controlled by regulating muscle activation and neural feedback gain. Although many attempts have been made to measure human multijoint arm stiffness, only a few researchers have tackled the contribution of multiple muscle activation to multijoint stiffness (Flash and Mussa-Ivaldi 1990; Tsuji et al. 1996). Linear relationships between single-muscle activation and the corresponding muscle stiffness or single-joint stiffness have been reported (Cannon and Zahalak 1982; Joyce and Rack 1969; Kearney and Hunter 1990). The relationship observed between single-muscle and single-joint stiffness possibly can be extended to the relationship between multijoint stiffness and multiple-muscle activation. Accordingly, each term of joint-stiffness matrix can be decomposed into the stiffness components contributed by each muscle's stiffness, which we will call 'effective muscle stiffness."

The contributions of monoarticular and biarticular muscle stiffness to each term of the stiffness matrix is worth examining because they may play an important role in altering the shape or orientation of the stiffness ellipse, which represents an elastic force that resists displacement of the hand in any direction in the horizontal plane. It has been reported that the stiffness ellipse size can vary while shape or orientation cannot (Dolan et al. 1993; Flash and Mussa-Ivaldi 1990; Hogan 1985; Mussa-Ivaldi et al. 1985; Tsuji et al. 1995). Lacquaniti, Carrozzo, and Borghese (1993), however, showed that stiffness can be modified during catching tasks, suggesting that stiffness may change with environmental interaction. In addition, Gomi and Osu (1998) showed that the stiffness ellipses also can be altered voluntarily by regulating muscle activation in isometric contraction tasks. The shape and orientation of a hand-stiffness ellipse is influenced most strongly by the ratio of single-joint stiffness (diagonal terms of the joint-stiffness matrix) and cross-joint stiffness (off-diagonal terms of the joint-stiffness matrix that govern interjoint-interaction between the shoulder and elbow joints). Because single-joint stiffness is composed of both monoarticular and biarticular effective muscle stiffness, whereas cross-joint stiffness is only composed of biarticular effective muscle stiffness, the regulation of monoarticular and biarticular muscles activation should play an important role in regulating the shape of the stiffness ellipse.

Stiffness ellipse alteration, however, was found to be limited in isometric tasks because the magnitude of cross-joint stiffness was always much smaller than the magnitude of single- 
joint stiffness (Gomi and Osu 1998). On the contrary, during movement, drastic changes in the elliptical orientations were observed, especially in the presence of external constraints such as a handle that can only be moved in one direction along the $x$ axis (Gomi and Kawato 1995, 1996a). In the middle of this constrained movement, the magnitude of the elbow singlejoint stiffness became as small as the magnitude of the crossjoint stiffness. This indicates that the regulation of monoarticular and biarticular muscles during movement is quite different from that in isometric task. These different formations in the joint-stiffness matrix between static and dynamic tasks suggest adaptive changes in muscle regulation according to the task requirements.

This paper examines the relation between joint stiffness and muscle activities during multijoint static force-regulation tasks in the horizontal plane at shoulder level. We predict the joint stiffness from the surface electromyographic (EMG) signals of six muscles that correspond to idealized muscles in the twolink arm model by using the constrained least-square-error regression method. Using the estimated parameters, we examine the muscle-regulation mechanisms, especially, the regulation of biarticular muscles, in the light of task dependency. The estimation of such parameters is practical because we can predict stiffness matrix from surface EMG signals without directly measuring them. Note that the analysis of the spatial characteristics of stiffness was presented elsewhere (Gomi and Osu 1998).

\section{METHODS}

\section{Experiment}

Four subjects, aged 23-34 yr old, took part in this study (subjects $A$ and $B$ : right-handed males, subject $C$ : ambidextrous female, subject $D$ : right-handed female). The subjects were seated on a chair in front of the parallel link drive air-magnet floating manipulandum (PFM) with their shoulders fixed to the back of the chair by straps (see Fig. 1). The handle position of the PFM was controlled by a high gain position servo. The right forearm of the subjects was fixed to a molded plastic cuff tightly coupled to the handle of the PFM and supported in the direction of gravity by a beam. The wrist joint of the subjects was fixed by the cuff and only the exertion of the shoulder and elbow joint torque in the horizontal plane were permitted. The right hand of each subject was kept at a specified position by the PFM during the experiment.

A computer monitor behind the PFM displayed the current force vector applied by the hand to the handle and a small circle indicating the target force. Subjects were asked to keep the head of the force vector on the target during each experimental set to preserve a constant external force. The current rectified and filtered surface EMG signals (moving average: $0.5 \mathrm{~s}$ ) of six muscles were displayed in a bar graph (current EMG bar graph) during the experimental set. A reference line was marked on the current EMG bar graph. The reference line was the rectified and filtered surface EMG signals (moving average: $0.5 \mathrm{~s}$ ) of six muscles that was determined by requesting target force exertion before each experimental set. The subjects were asked to keep the current EMG bar graph the same as the reference line on the EMG bar graph so that the muscle activities would be constant during each set.

The hand of each subject was placed in the following five positions: proximal center $(\mathrm{PC}:[x, y]=[0.0,0.35] \mathrm{m})$, middle center $(\mathrm{MC}:[0.0$, $0.45] \mathrm{m}$ ), distal center (DC: $[0.2,0.55] \mathrm{m})$, proximal left (PL: $[-0.2$, $0.35] \mathrm{m}$ ), and proximal right (PR: $[0.2,0.35] \mathrm{m}])$. Two types of tasks were given to the subjects.

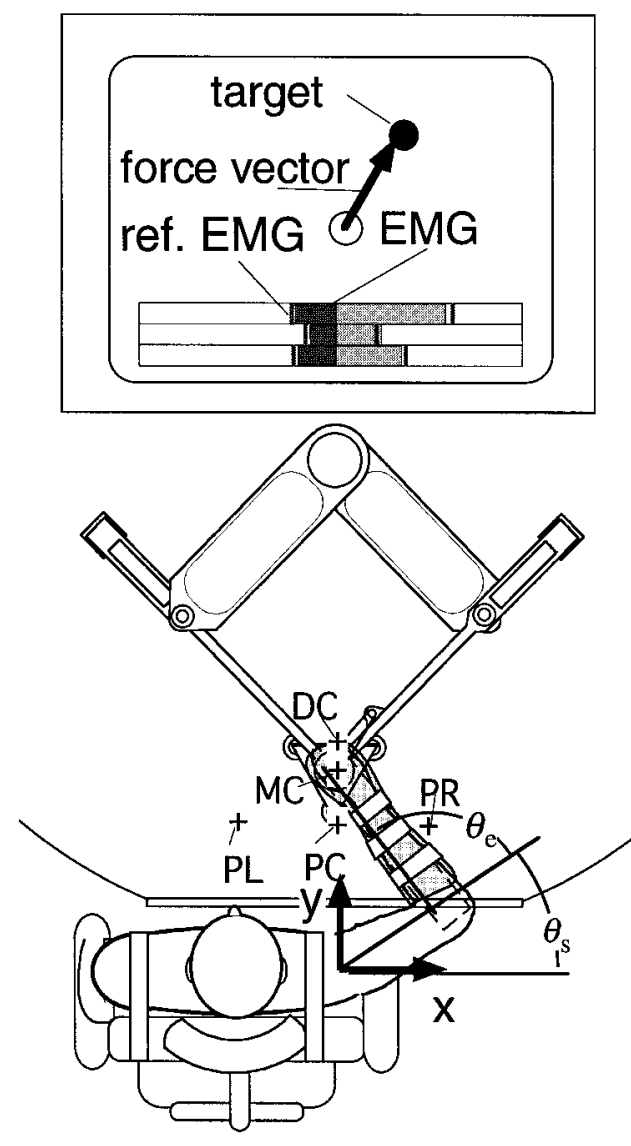

FIG. 1. Parallel link drive air-magnet floating manipulandum (PFM) system and experimental setup for measuring human arm mechanical impedance. $x$ axis indicates right direction and $y$ axis indicates frontal direction away from body. Origin is shoulder position. Force vector in horizontal plane was displayed on the computer monitor. Electromyogram (EMG; rectified and averaged) levels of 6 muscles were shown by bar graph to maintain muscle activation level in each experimental set.

FORCE-REGULATION TASKS WITHOUT COCONTRACTION. The subjects were asked to produce a specified force $(5,10,15$, or $20 \mathrm{~N})$ in a specified direction [8 directions $(5 \mathrm{~N})$ or 16 directions $(10,15,20 \mathrm{~N})$ in the hand's $x-y$ plane at even intervals] without cocontraction.

POSTURE MAINTENANCE AND FORCE-REGULATION TASKS WITH DIFFERENT COCONTRACTIONS. The subjects were asked to maintain different contractions while producing, or not producing, an external force. The specified tasks were to keep the current force vector $0: 1$ ) without cocontraction, 2) with a quarter cocontraction, 3) half, 4) full cocontraction, 5) with cocontraction only in the shoulder, 6) with cocontraction only in the elbow, or 7) to produce a $5-\mathrm{N}$ force in a specified direction (4 directions in the horizontal plane) with moderate cocontraction.

The tasks requested of each subject were as follows. All subjects: PC $(5,10,15 \mathrm{~N})$; DC $(10 \mathrm{~N}) ; 1-6$ at PC, MC, DC, and 7 at PC. Subject $A$ was also: PC $(20 \mathrm{~N}), \mathrm{MC}(5,10,15 \mathrm{~N}), \mathrm{PL}, \mathrm{PR}(10 \mathrm{~N}), 1-6$ at PL, PR, and 7 at MC. Subject B was also PC (20 N) and MC (10 $\mathrm{N})$; subject $D$ was also MC $(10 \mathrm{~N})$.

To estimate impedance parameters, the hand was pushed slightly and pulled back $(6-8 \mathrm{~mm})$ within a brief period $(0.3 \mathrm{~s})$ in eight randomized directions ( 3 trials for each of 8 directions in each experimental set). The rising time of the positional displacement (duration between perturbation onset and the peak displacement) was $0.17 \mathrm{~s}$. The displacement amplitude was almost the same for all tasks due to the positional perturbation. The subjects were asked not to intervene voluntarily during the perturbation, and all visual feedback 
on the monitor (i.e., current force vector and current EMG bar graph) was frozen. The hand position, force, and EMG signals were recorded. The applied external forces were decomposed into arm dynamics and muscle-generated force. Details of the arm impedance estimation method are provided in elsewhere (Gomi and Kawato 1995, 1996a,b, 1997; Gomi and Osu 1998).

Surface EMG signals were recorded from the muscles that corresponded to the six muscles in the model. For shoulder monoarticular muscles, activities in the pectoralis major (flexor) and the posterior deltoid (extensor) were measured. For elbow monoarticular muscles, activities in the brachioradialis (flexor) and the lateral head of triceps brachii (extensor) were measured. For biarticular muscles, activities in the biceps brachii (flexor) and the long head of triceps brachii (extensor) were measured. The EMG signals were recorded by using pairs of silver-silver chloride surface electrodes in a bipolar configuration. Each signal was filtered [cutoff frequency: $25 \mathrm{~Hz}$ (low), 1,500 $\mathrm{Hz}$ (high)] and sampled at 2,000 Hz. The EMG signals measured on different days were normalized and scaled (see APPENDIX A). The normalized and scaled EMG signals then were rectified and averaged for a period of $0.4 \mathrm{~s}$ before perturbation, which we refer to as the "EMG levels." The EMG levels were used for parameter estimation.

\section{Estimation of joint-stiffness from EMG levels}

A human arm on the horizontal plane can be modeled as a two-link manipulator with four monoarticular muscles and two biarticular muscles. The relation between joint-stiffness and EMG levels was estimated by the arm model and a least-square-error method as described in the following text.

Joint stiffness $\mathbf{R}$ is expressed as a differential operator that relates the small variation of joint torque $\tau$ to the small angular displacement q. The relation between joint stiffness $\mathbf{R}$ and muscle stiffness $\mathbf{S}$ can be defined as follows (see APPENDIX B for details).

$$
\begin{gathered}
\mathbf{R}=-\frac{\partial \tau}{\partial \mathbf{q}}=\mathbf{A} \cdot \mathbf{S} \cdot \mathbf{A}^{T} \\
\mathbf{R}=\left(\begin{array}{cc}
R_{\mathrm{ss}} & R_{\mathrm{cj}} \\
R_{\mathrm{cj}} & R_{\mathrm{ee}}
\end{array}\right) \\
\mathbf{A}=\left(\begin{array}{cccccc}
d_{1} & -d_{2} & 0 & 0 & d_{5} & -d_{6} \\
0 & 0 & d_{3} & -d_{4} & d_{7} & -d_{8}
\end{array}\right) \\
\mathbf{S}=\operatorname{diag}\left[s_{1}, s_{2}, s_{3}, s_{4}, s_{5}, s_{6}\right]=\operatorname{diag}\left[p_{1} u_{1}+s_{01}, \ldots, p_{6} u_{6}+s_{06}\right]
\end{gathered}
$$

Here $d_{\mathrm{i}}$ denotes the moment arm. We assume that muscle stiffness $s_{\mathrm{i}}$ is proportional to individual muscle activity $u_{\mathrm{i}}$ (Joyce and Rack 1969; Milner et al. 1995). $s_{1}$ and $s_{2}$ denote stiffness of shoulder monoarticular flexor and extensor, $s_{3}$ and $s_{4}$ denote stiffness of elbow monoarticular flexor and extensor, and $s_{5}$ and $s_{6}$ denote stiffness of biarticular flexor and extensor, respectively. $p_{\mathrm{i}}$ denotes positive constant coefficients, and $s_{0 \mathrm{i}}$ denotes the intrinsic stiffness value when the muscle is inactive. Off-diagonal terms of the joint stiffness matrix (cross-joint stiffness), $R_{\mathrm{cj}}$, are assumed to be equal, although the reflex components possibly may produce asymmetry in a stiffness matrix (Hogan 1985; Mussa-Ivaldi et al. 1985). Substituting Eqs. 2-4 into $E q$. 1, each term of joint stiffness $\mathbf{R}$ can be expressed as follows (see APPENDIX B for details).

$$
\begin{gathered}
R_{\mathrm{ss}}=a_{1} u_{1}+a_{2} u_{2}+a_{5} u_{5}+a_{6} u_{6}+b_{1} \\
R_{\mathrm{cj}}=a_{9} u_{5}+a_{10} u_{6}+b_{2} \\
R_{\mathrm{ee}}=a_{3} u_{3}+a_{4} u_{4}+a_{7} u_{5}+a_{8} u_{6}+b_{3}
\end{gathered}
$$

$a_{\mathrm{i}} u_{\mathrm{j}}$ denotes effective muscle stiffness of the $j$ th muscle, that is, the contribution of $j$ th muscle activation to the corresponding joint stiffness. When the arm posture is fixed, the $a_{\mathrm{i}}$ and $b_{\mathrm{i}}$ are constant as long as the moment arm does not change with the muscle activation levels. For different postures, they are also constant if the moment arm can be assumed to be constant (Winters 1990). It follows that each term of the joint stiffness matrix $\mathbf{R}$ can be expressed as a linear sum of muscle activities $u_{\mathrm{i}}$. In addition, the following constraints are used by the least-square-error method.

$$
\begin{gathered}
a_{\mathrm{i}}>0, b_{\mathrm{i}}>0 \\
a_{9}{ }^{2}=a_{5} a_{7}, a_{10}{ }^{2}=a_{6} a_{8}
\end{gathered}
$$

Because moment arm $d_{\mathrm{i}}$ (in $E q$. 3), constant coefficients $p_{\mathrm{i}}$ (in $E q .4$ ), and intrinsic muscle stiffness $s_{0 \mathrm{i}}$ (in $E q .4$ ) are all positive, $E q .6$ can be used as a constraint. Equation 7 can be used as another constraint because the biarticular effective muscle stiffness of single-joint stiffness $\left(a_{5} u_{5}\right.$ and $a_{6} u_{6}$ of $R_{\mathrm{ss}} ; a_{7} u_{5}$ and $a_{8} u_{6}$ of $\left.R_{\mathrm{ee}}\right)$ and those of cross-joint stiffness $\left(a_{9} u_{5}\right.$ and $a_{10} u_{6}$ of $\left.R_{\mathrm{cj}}\right)$ share the same moment arm (see $E q . B 5$ in APPENDIX B).

Assuming that muscle activities $u_{\mathrm{i}}$ are proportional to EMG levels, we examined whether we could reconstruct the joint stiffness matrix $\mathbf{R}$ from measured EMG levels. A constrained optimization method (the sequential quadratic programming method in MATLAB software) was used to estimate the parameters that satisfied Eqs. 6 and 7. The averaged values of off-diagonal terms of the measured joint stiffness matrix were used as cross-joint stiffness $R_{\mathrm{cj}}$ because the stiffness matrix under static conditions (i.e., posture maintenance and force-regulation tasks) was nearly symmetric (Gomi and Osu 1998; Mussa-Ivaldi et al. 1985; Tsuji et al. 1995) as assumed in the model.

We obtained preferred hand-force direction for each muscle by calculating the maximum correlation angle between the positive range of the cosine function $(-\pi / 2 \sim \pi / 2)$ and the EMG levels under force-regulation tasks in all 16 directions.

\section{R E S U L T S}

\section{Regression performance}

Because a nonlinear increase in EMG signal with muscle force has been reported in high muscle activation, we separated the data according to the contraction level (Basmajian and De Luca 1985). We performed the estimation using the following three data groups. The first group contains data under forceregulation tasks without cocontraction and rest condition (a), both at the proximal center posture. The second group contains data under the same condition as in the first group but at all postures. The third group contains data under posture maintenance and force-regulation tasks with different amounts of cocontraction and the maximum hand force in the force-regulation tasks, (subjects $A$ and $B: 20 \mathrm{~N}, 16$ directions; subjects $C$ and $D: 15 \mathrm{~N}, 16$ directions).

The correlation coefficients between the measured and reconstructed stiffness for each subject were 0.966, 0.951, 0.966, and 0.939 for the first data group. The correlation coefficients for the second data group were $0.951,0.932,0.921$, and 0.879 . The deterioration in estimation accuracy compared with the first data group might be ascribed to the difference in moment arms among the postures (van Zuylen et al. 1988). The correlation coefficients for the third data group were 0.943, 0.933, 0.940 , and 0.931 . Figure 2 compares each term of the measured and reconstructed stiffness values of subject $A$ using the first data group. The estimation of single-joint stiffness was better than that of cross-joint stiffness for all subjects.

Table 1 summarizes the estimated parameters and their $90 \%$ confidence intervals as well as the ratios of the confidence intervals to the estimated parameters for the three data groups (subject A). The parameters are different for the three data groups. The differences between the first and third data groups 


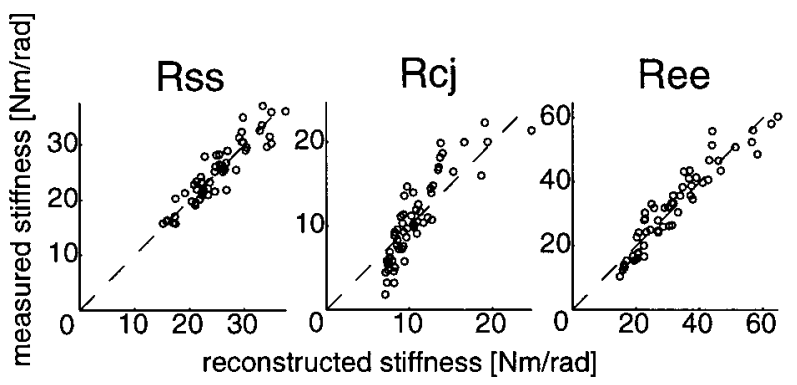

FIG. 2. Regression results using data under force-regulation tasks without cocontraction at proximal posture for subject A. Horizontal axis denotes reconstructed stiffness and vertical axis denotes measured stiffness. Left: shoulder-joint stiffness; middle: cross-joint stiffness; right: elbow-joint stiffness.

and between the second and third data groups are conspicuous compared with those between the first and second groups. The parameter difference cannot be ascribed to the different moment arms among the postures because both the second and third data group include data from all postures. Three possible explanations could account for these differences. The first is that conducting the EMG recordings on different days may have resulted in the failure of daily normalization. The second is that there may have been contributions by muscles other than those measured in the present experiment. The third is that there actually may be nonlinearity between the EMG levels and muscle stiffness. Because the normalization appears to be accurate as indicated by the values of correlation coefficients shown in APPENDIX A, only the second and third reasons appear feasible. Parameters $a_{5}, a_{7}$, and $a_{9}$, which are related to the biarticular flexor (biceps brachii), were considerably smaller under cocontraction than under force regulation for three subjects (subjects $A, B$, and $C$ ). The difference in parameters cannot be ascribed to the nonlinear contribution of other muscles (the 2nd reason) because there is only one biarticular flexor in the human arm. The nonlinear contribution of different heads of the biceps could hardly explain the parameter difference as well because only small differences have been reported between the activities of two heads of the biceps during isometric contraction (Basmajian and De Luca 1985). The difference suggests that the contribution of the biceps'
EMG levels to the biarticular flexor muscle stiffness $\left(p_{5}\right.$ in $E q$. 4 ) is decreased when the biceps are strongly activated.

\section{Relation between effective muscle stiffness and joint stiffness}

Figure $3 A$ shows the relation between joint stiffness and effective muscle stiffness $a_{\mathrm{i}} u_{\mathrm{j}}$ (EMG levels weighted by their corresponding estimated parameters) in tasks without cocontraction for subject $A$. All the other subjects showed a similar tendency. A sign was given to each joint-stiffness value according to the sign of the joint torque (a positive sign was given to flexor torque) related to that stiffness. Figure $3 A$, top, shows the relation between $R_{\mathrm{ss}}$ and the shoulder monoarticular effective muscle stiffness. The sign for the shoulder torque was given to $R_{\mathrm{ss}}$. Figure $3 A$, middle, shows the relation between $R_{\mathrm{cj}}$ and biarticular effective muscle stiffness. The sign for the elbow torque was given to $R_{\mathrm{cj}}$. Figure $3 A$, bottom, shows the relation between $R_{\mathrm{ee}}$ and the elbow monoarticular effective muscle stiffness. The sign for the elbow torque was given to $R_{\mathrm{ee}}$.

When muscles are working as agonists, that is, when the joint torque is positive for flexor muscles and when the joint torque is negative for extensor muscles, a strong correlation can be observed between effective muscle stiffness and joint stiffness. The relation is roughly linear, but in some cases, especially in the biceps brachii in subjects $A$ and $B$, an accelerating increase in effective muscle stiffness can be observed when muscles are strongly activated by high joint torque (see the panels at the right column of the second row in Fig. 3A). These responses were not so clear for subjects $C$ and $D$, which is possibly because they did not execute the tasks with the 20 $\mathrm{N}$ hand force. This nonlinearity in the biarticular flexor is one of the reasons of lower reliability in predicting cross-joint stiffness compared with that of single-joint stiffness.

Even when muscles are working as antagonists, a correlation can often be found between joint stiffness and effective muscle stiffness, especially in the elbow monoarticular muscles and the biarticular flexor. The slope is, however, lower when muscles are working as antagonists than when they are working as agonists. This observation indicates that a part of the net joint stiffness in force-regulation tasks can be ascribed to antagonist

TABLE 1. Estimated parameters, their $90 \%$ confidence intervals, and the ratios of confidence intervals to estimated parameters for subject $A$

\begin{tabular}{|c|c|c|c|c|c|c|c|c|c|}
\hline & \multicolumn{6}{|c|}{ Force Regulation } & & & \\
\hline & \multicolumn{3}{|c|}{ Proximal Center Posture } & \multicolumn{3}{|c|}{ All Postures } & \multicolumn{3}{|c|}{ Cocontraction } \\
\hline & $\mathrm{a}$ & $\mathrm{b}$ & $\mathrm{c}$ & $\mathrm{a}$ & $\mathrm{b}$ & $\mathrm{c}$ & $\mathrm{a}$ & $\mathrm{b}$ & $\mathrm{c}$ \\
\hline$a_{1}$ & 39.97 & 7.28 & 0.18 & 37.12 & 4.04 & 0.11 & 45.96 & 9.62 & 0.21 \\
\hline$a_{2}$ & 92.19 & 12.12 & 0.13 & 79.85 & 6.74 & 0.08 & 89.35 & 17.59 & 0.20 \\
\hline$a_{3}$ & 109.97 & 28.60 & 0.26 & 125.42 & 18.95 & 0.15 & 179.52 & 39.35 & 0.22 \\
\hline$a_{4}$ & 58.56 & 42.49 & 0.73 & 59.18 & 23.90 & 0.40 & 95.96 & 49.65 & 0.52 \\
\hline$a_{5}$ & 74.44 & 18.91 & 0.25 & 78.61 & 13.04 & 0.17 & 14.68 & 9.48 & 0.65 \\
\hline$a_{6}$ & 15.38 & 10.49 & 0.68 & 13.11 & 6.93 & 0.53 & 38.08 & 19.44 & 0.51 \\
\hline$a_{7}$ & 179.72 & 60.18 & 0.33 & 124.85 & 35.82 & 0.29 & 48.61 & 19.91 & 0.41 \\
\hline$a_{8}$ & 34.99 & 52.44 & 1.50 & 40.58 & 29.03 & 0.72 & 23.08 & 76.90 & 3.33 \\
\hline$a_{9}$ & 115.66 & 22.01 & 0.19 & 99.07 & 16.41 & 0.17 & 26.71 & 13.98 & 0.52 \\
\hline$a_{10}$ & 23.20 & 10.97 & 0.47 & 23.07 & 7.65 & 0.33 & 29.65 & 27.22 & 0.92 \\
\hline$b_{1}$ & 13.65 & 1.30 & 0.10 & 13.56 & 0.71 & 0.05 & 13.71 & 1.95 & 0.14 \\
\hline$b_{2}$ & 6.84 & 1.04 & 0.15 & 6.64 & 0.68 & 0.10 & 7.92 & 2.41 & 0.30 \\
\hline$b_{3}$ & 14.55 & 2.11 & 0.14 & 12.81 & 1.23 & 0.10 & 9.72 & 3.07 & 0.32 \\
\hline
\end{tabular}

$\mathrm{a} \pm \mathrm{b}$, estimated parameters \pm their confidence intervals; $\mathrm{c}$, ratios of confidence intervals to estimated parameters (b/a). 
activation. Joint stiffness in force-regulation tasks is correlated strongly to joint torque (Gomi and Osu 1998). The present results indicate that coactivation (and hence stiffness) also increases as the joint torque increases even under relaxed conditions without instructions to cocontract. Coactivation un-
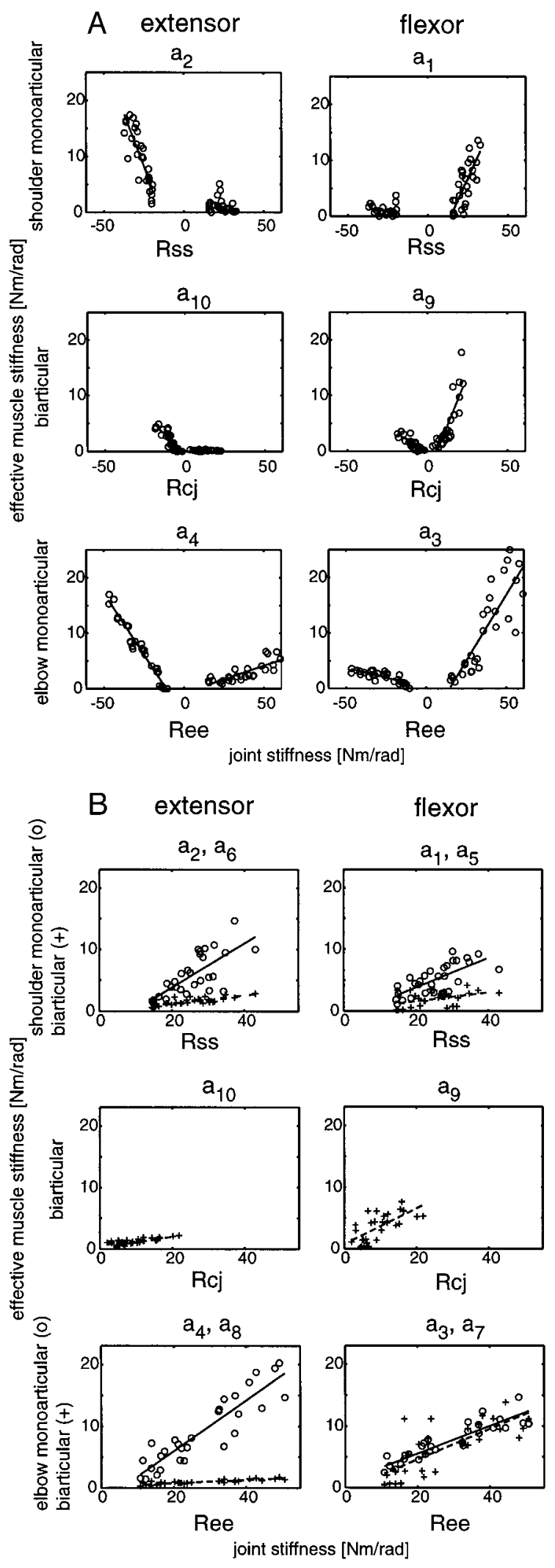

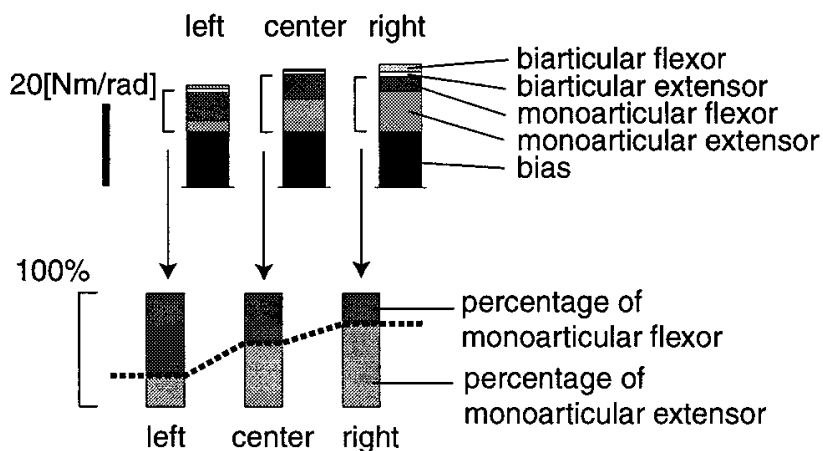

FIG. 4. Ratios between shoulder monoarticular flexor and extensor effective muscle stiffness in shoulder single-joint stiffness at proximal left, center, and right postures in posture maintenance task (e: shoulder cocontraction) for subject A. Top: contribution of each muscle stiffness to shoulder joint stiffness. Each bar is divided into gray-scaled regions, each denotes different muscle contribution. Bottom: ratios between monoarticular flexor and extensor effective muscle stiffness in shoulder joint stiffness at the corresponding postures.

der force-regulation tasks was reported to be observed in the sagittal plane (Flanders and Soechting 1990).

Figure $3 B$ shows the relation between joint stiffness and effective muscle stiffness in posture maintenance tasks with different levels of cocontraction. The panel arrangements are the same as in Fig. 3A. Open circles and solid line denote the monoarticular effective muscle stiffness and their regression lines, respectively. Plus signs and dashed line denote the biarticular effective muscle stiffness and their regression lines, respectively. In all muscles, the effective muscle stiffness linearly increased as the corresponding joint stiffness increased.

\section{Variations in effective muscle stiffness depending on posture and force direction}

In Fig. 4, the ratios between shoulder monoarticular flexor and extensor effective stiffness in shoulder single-joint stiffness are compared among proximal left, center, and right postures. Figure 4, top, represents the contribution of each muscle stiffness to shoulder joint stiffness in a posture maintenance task (e: shoulder cocontraction) for subject A; bottom represents the ratios between monoarticular flexor and extensor effective stiffness in shoulder joint stiffness at the corresponding postures. As shown in the figure, the muscles that contribute most to shoulder stiffness change with the posture. At proximal left, the monoarticular flexor contributes to shoulder

FIG. 3. A: relation between effective muscle stiffness and joint stiffness under force-regulation tasks without cocontraction for subject A. Horizontal axis denotes joint stiffness with sign given according to sign of joint torque related to that stiffness (positive: flexion torque). Vertical axis denotes rectified and averaged EMG levels weighted by corresponding parameters estimated using these data (effective muscle stiffness). Left: extensor muscles; right: flexor muscles. Top: relation between shoulder monoarticular effective muscle stiffness and shoulder stiffness with sign for shoulder torque. Middle: relation between biarticular effective muscle stiffness and cross-joint stiffness with sign for elbow torque. Bottom: relation between elbow monoarticular effective muscle stiffness and elbow stiffness with sign for elbow torque. Regression lines are drawn for significant $(P<0.01)$ correlation between joint stiffness and effective muscle stiffness. $B$ : relation between effective muscle stiffness and joint stiffness under posture maintenance tasks with different cocontraction for subject A. Panel arrangements are the same as in A. Open circles and solid line, monoarticular effective muscle stiffness and their regression lines. Plus signs and dashed line, biarticular effective muscle stiffness of and their regression lines. 
stiffness more than the monoarticular extensor. At proximal right, however, the reverse is true. At proximal center, both the monoarticular flexor and monoarticular extensor contribute to shoulder joint stiffness. These phenomena can be explained by the muscles' length-tension relation. At proximal left, the shoulder monoarticular flexor is shortened and the shoulder monoarticular extensor lengthened compared with the positions of proximal center or proximal right, so there is necessarily more activation in the flexor and less activation in the extensor to hold the arm at the specified posture. On the other hand, when the shoulder monoarticular flexor is lengthened and the shoulder monoarticular extensor shortened at proximal right, there is necessarily less activation in the flexor and more activation in the extensor to keep the arm at the specified posture. If muscle stiffness is proportional to muscle activation, the contribution of the shoulder monoarticular flexor and extensor to shoulder stiffness is expected to vary with postures.

Identical explanations are possible for the contribution difference of elbow monoarticular muscles between proximal center and distal center for subject $A$. At proximal center, the elbow monoarticular flexor is shortened and the elbow monoarticular extensor lengthened compared with the distal center position. More activation in the flexor and less activation in the extensor was observed at the proximal position compared with the distal position, which is also consistent with the lengthtension relationship.

Figure 5 shows the variations of effective muscle stiffness against hand-force directions for subject $A$ (left: extensor, right: flexion). Figure $5, A$ and $B$, shows the effective muscle stiffness values of shoulder monoarticular (solid thick curve) and biarticular muscles (dotted thick curve) in the shoulder joint stiffness for $15 \mathrm{~N}$ forces. $C$ and $D$ and $E$ and $F$ show corresponding effective muscle stiffness in the cross-joint and elbow joint stiffness, respectively. The corners of the dash-dot thin octagons in the first and second rows represent normalized absolute shoulder torque plotted against the hand-force direction (torque vector). The corners of the dashed thin octagons in the second and third rows represent normalized absolute elbow torque plotted against the hand-force direction. The octagons on the left denote extension torque, and those on the right denote flexion torque. In the hand-force direction where the shoulder torque vector and elbow torque vector overlap (see the 2nd row), the shoulder and elbow torque are in the same direction [both in extension direction $(C)$ or both in flexion direction $(D)$ ]. As shown $(A$ and $B)$, biarticular muscles contribute to single-joint stiffness (dotted thick curves) more extensively in the flexion directions $(8-12)$ than in the extension directions (1-4, and 16). Biarticular muscles contribute to elbow joint stiffness more strongly than they do to shoulder joint stiffness [see dotted thick curves in graphs $(A, B, E$, and $F)$ ]. In general, stiffness mainly is composed of agonist activations, but in the elbow joint, antagonist activations also contribute to stiffness (e.g., the elbow monoarticular flexor and extensor, biarticular flexor). The contribution of antagonists was observed most frequently in the elbow joint in force directions $1-6$, where the elbow torque was negative. All the other subjects showed a similar tendency.

In force directions $5,6,7,13,14$, and 15 , the directions of the shoulder torque and elbow torque oppose each other or either elbow torque alone, or shoulder torque alone, is exerted. The activation of biarticular muscles should not
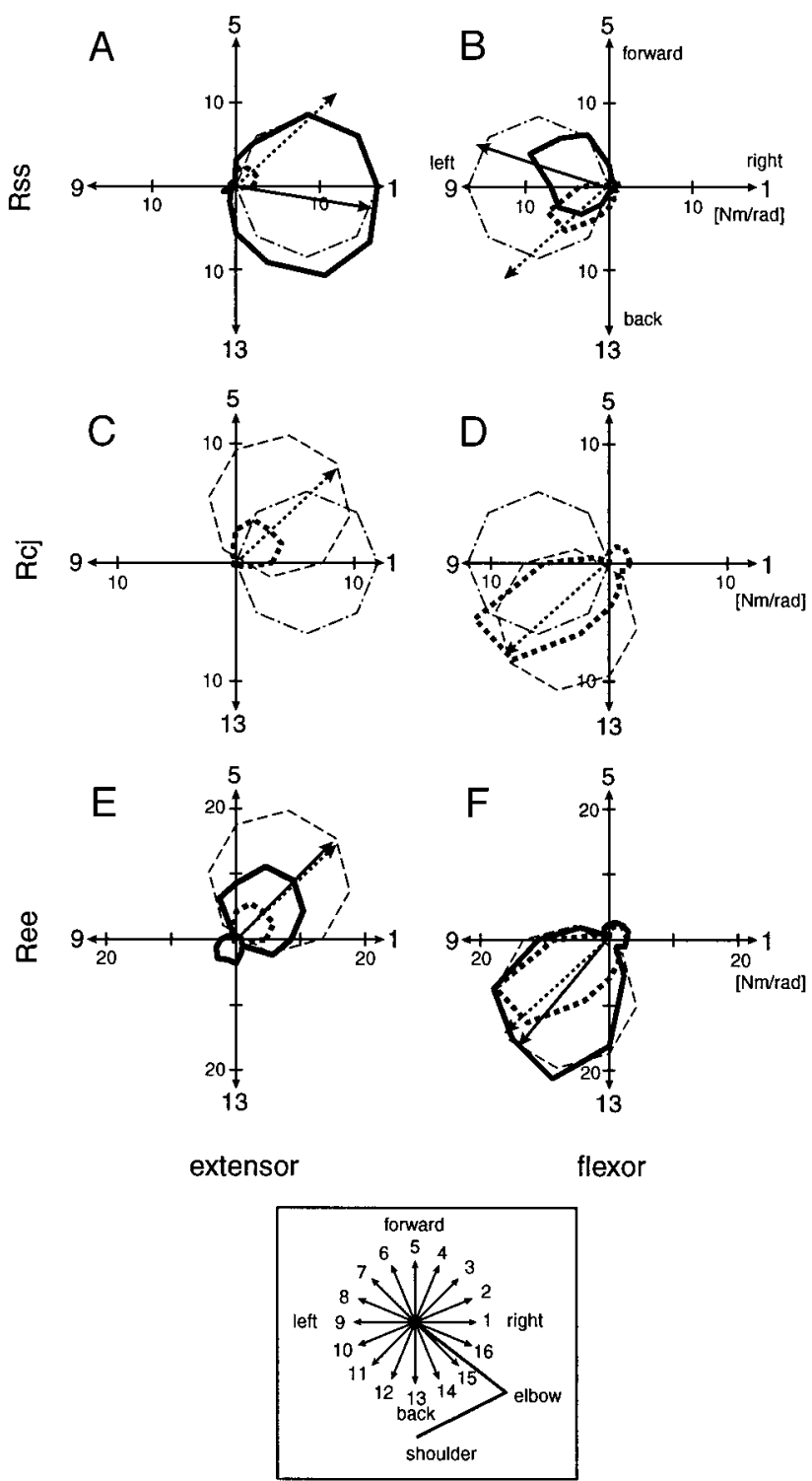

FIG. 5. Variations of effective muscle stiffness against hand-force direction under force-regulation tasks without cocontraction $(15 \mathrm{~N})$ for subject $A$. Bottom: hand-force directions. Left: extensor effective muscle stiffness; right: flexor effective muscle stiffness. First, 2nd, and 3rd rows show shoulder, cross-joint, and elbow stiffness respectively. Solid thick curves: monoarticular muscle contribution, dotted: biarticular muscle contribution. Corners of dashdot thin octagons in left and right columns denote normalized absolute shoulder extension and flexion torque respectively (shoulder torque vectors). Corners of dashed thin octagons in left and right columns denote normalized absolute elbow extension and flexion torque respectively (elbow torque vectors). Shoulder and elbow torques have same direction at the hand-force direction where the shoulder and elbow torque vectors overlap. Solid arrows denote preferred force direction of monoarticular muscles at $15 \mathrm{~N}$ force, dotted arrows denote preferred force direction of biarticular muscles at $15 \mathrm{~N}$ force.

effective in such directions (Karst and Hasan 1991). However, biarticular muscles, especially the biarticular flexor, were activated in some of these directions $(13,14$, and 15; Fig. 5D). The activation of the shoulder monoarticular extensor would have been greater than the activation needed to generate the extension torque that meets the task requirements because the shoulder flexion torque generated by the biarticular flexor was canceled out. 
TABLE 2. Mean and standard deviation of absolute difference in preferred direction between monoarticular muscles and biarticular muscles

\begin{tabular}{lcc}
\hline \hline & $\begin{array}{c}\text { Shoulder Mono-/ } \\
\text { Biarticular, deg }\end{array}$ & $\begin{array}{c}\text { Elbow Mono-/ } \\
\text { Biarticular, deg }\end{array}$ \\
\hline $\begin{array}{l}\text { Subject } A \\
\text { Extensor }\end{array}$ & $52 \pm 3.0$ & \\
$\quad$ Flexor & $61 \pm 1.7$ & $2 \pm 0.6$ \\
Subject $B$ & $34 \pm 4.0$ & $7 \pm 6.0$ \\
$\quad$ Extensor & $47 \pm 1.0$ & $24 \pm 1.5$ \\
Flexor & $44 \pm 0.7$ & $5 \pm 1.7$ \\
Subject $C$ & $65 \pm 4.9$ & $10 \pm 6.4$ \\
Extensor & & $1 \pm 1.4$ \\
Flexor & $41 \pm 2.1$ & $28 \pm 0.7$ \\
Subject $D$ & $59 \pm 2.1$ & $1 \pm 0.0$ \\
Extensor & & \\
Flexor & & \\
\hline
\end{tabular}

The solid and dotted arrows in the graphs denote the preferred force direction (defined in METHODS) of monoarticular and biarticular muscles at $15 \mathrm{~N}$ force, respectively. The preferred force direction of the elbow and shoulder monoarticular muscles (solid arrows) roughly corresponds to the force direction where the maximum torque is required at the corresponding joint. However, the biarticular muscles' preferred force direction (dotted arrows) was not located in the middle of the shoulder and elbow monoarticular muscles' preferred force direction (Fig. 5, $C$ and $D$ ), but closer to the elbow monoarticular muscles' preferred direction (Fig. 5, $E$ and $F$ ). Table 2 shows the absolute difference of the preferred direction between monoarticular muscles and biarticular muscles. For all subjects, the differences between shoulder monoarticular muscles and biarticular muscles were larger than those between elbow monoarticular muscles and biarticular muscles. The biarticular flexor's preferred direction was almost the same as the elbow monoarticular flexor's preferred direction. The mean and the standard deviation of correlation coefficients between EMG levels and the fitted cosine function at the preferred direction for four subjects were as follows: 0.99 and 0.013 for shoulder monoarticular muscles, 0.95 and 0.039 for biarticular muscles, and 0.96 and 0.029 for elbow monoarticular muscles.

In force-regulation tasks $(5 \mathrm{~N})$ with cocontraction, both monoarticular muscles and biarticular muscles were activated in all four directions. The shoulder joint stiffness was high in the force direction toward or away from the shoulder joint (directions 5 and 13), although it was low in those tasks without cocontraction (directions 5 and 13 in Fig. 5). Although the directional differences in joint stiffness were decreased by cocontraction, the contribution of different muscles to stiffness differed with the force direction. Without cocontraction, the stiffness ellipses in directions 5 and 13 were thinner than those in other directions, i.e., the hand is more compliant in a direction perpendicular to the force (Gomi and Osu 1998; McIntyre et al. 1996). Cocontraction can stabilize the hand even in these compliant pushing or pulling force directions.

\section{Difference in contribution of biarticular and monoarticular muscles to single-joint stiffness}

Next, we examined the contribution of each muscle to single-joint stiffness. Figure $6 \mathrm{~A}$ shows the relationships between biarticular and monoarticular effective muscle stiffness in shoulder and elbow single-joint stiffness (subject $C$, forceregulation tasks without cocontraction). Because the preferred directions of elbow mono- and biarticular muscles were similar to each other, as observed in the preceding section, the biarticular and monoarticular effective muscle stiffness at elbow is expected to be highly correlated to each other. The mean \pm SD of correlation coefficients between them for four subjects were $0.78 \pm 0.15$ for elbow extensor, $0.84 \pm 0.085$ for elbow flexor. Biarticular muscles contribute little to shoulder joint stiffness but contribute much to elbow joint stiffness for all subjects. The slopes of regression lines between the elbow mono- and biarticular effective muscle stiffnesses were greater than one for both extensor and flexor (mean \pm SD for 4 subjects, extensor: $3.00 \pm 1.24$, flexor: $1.80 \pm 0.83$ ), indicating that monoarticular muscle contributions to joint stiffness were greater than biarticular muscle contribution. On the other hand, in the tasks with cocontraction (Fig. 6B), the slope of the regression line was decreased (mean \pm SD: $1.15 \pm 0.62$ ) compared with that in the tasks without cocontraction. The
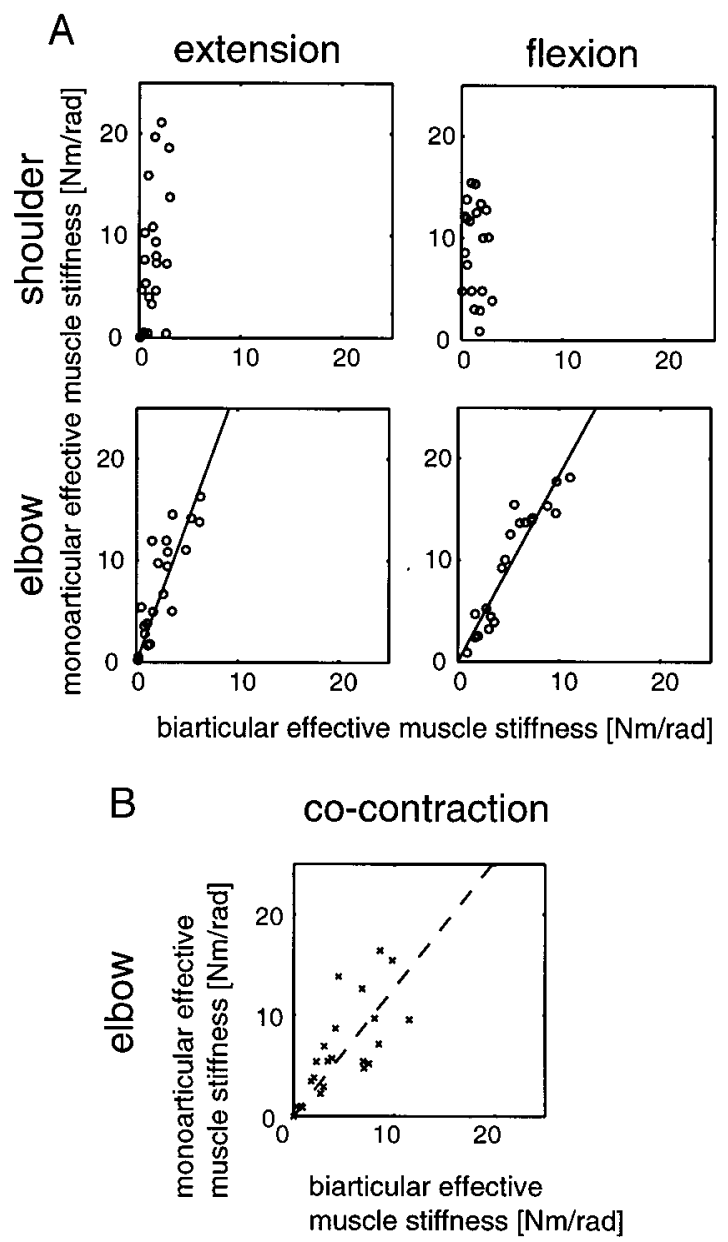

FIG. 6. A: ratio between contributions of biarticular and monoarticular muscles to single-joint stiffness under force-regulation tasks without coontraction at the proximal center posture for subject $C$. Horizontal axis denotes biarticular effective muscle stiffness, vertical axis denotes monoarticular effective muscle stiffness. Top: shoulder stiffness; bottom: elbow stiffness. Left: stiffness in extension; right: stiffness in flexion. Regression lines for elbow stiffness are shown (correlation coefficients for shoulder stiffness were low). $B$ : ratio between contributions of biarticular and elbow monoarticular muscles to elbow single-joint stiffness under cocontraction tasks for subject $C$. 
decrease in the slope indicates that the contribution of biarticular muscles increased in cocontraction tasks, which resulted in increasing posture stability.

\section{Reflex contribution to stiffness}

It has been reported that stretch reflex mainly responds to stretching velocity, thus stretch reflex contributes little to stiffness, especially if the stretch is small and quick (Kearney and Hunter 1983; Matthews and Stein 1968; Stein et al. 1995). However, muscle spindle sensitivity seems to change with perturbation amplitude and/or velocity (Kearney and Hunter 1983). To examine the effect of reflex components on stiffness estimation in the current setup, we observed the correlation between the rectified EMG signals of elbow monoarticular muscles and the kinematic components of elbow joint (position, velocity, and acceleration) during perturbation (0.6-s period beginning $0.2 \mathrm{~s}$ before perturbation onset). Both in elbow flexion (force directions 9-13) and extension (force direction 1-5) tasks, the EMG signals of elbow single-joint flexor muscles of the four subjects were better correlated to perturbation velocity $(0.18 \pm 0.024$ for flexion, $0.16 \pm 0.032$ for extension $)$ than to position $(0.018 \pm 0.016$ for flexion, $-0.040 \pm 0.016$ for extension) or acceleration $(0.033 \pm 0.038$ for flexion, $0.085 \pm 0.023$ for extension). These results suggests that, at the perturbation amplitude and velocity examined here, reflex components contribute little to stiffness; they mainly contribute to viscosity.

\section{I S C U S S I O N}

\section{Joint stiffness predicted from EMG signals}

The roughly linear relationship of muscle activation and muscle stiffness has been observed in human muscles and in isolated cat muscles (Cannon and Zahalak 1982; Joyce and Rack 1969; Kearney and Hunter 1990). On the basis of these observations, we tried to extend this linear characteristic to model the relationships between the EMG level of multiple muscles and multijoint stiffness. The success of the model for various conditions indicates that our simple model can predict joint stiffness from EMG measurements and permits the contribution of each muscle to joint stiffness to be estimated. The success of the model also confirmed the assumption that muscle stiffness is proportional to EMG level.

However, results in Fig. 3 suggest that there is nonlinearity in the relation between the rectified averaged surface EMG levels and joint stiffness, especially in biceps brachii, which degraded estimation accuracy. Joint stiffness tends to saturate when the muscles are strongly activated by cocontraction or by a high joint torque. According to Basmajian and De Luca (1985), the relationship between EMG signal amplitude and force output for small hand muscles such as the first dorsal interosseous and the adductor pollicis always has been found to be quasilinear, whereas in larger muscles such as the biceps brachii, triceps brachii, deltoid, and soleus, the signal amplitude increases more than the force. Such nonlinearity may be explained partially by a change in the regulation of muscle tension according to the muscle tension magnitude. At a low contraction level, the force mainly is increased by motor unit recruitment, whereas at a higher contraction level, the rising firing frequency increases the muscle tension (Kanosue et al.
1979). The effect of muscle fatigue, another possible factor of the nonlinearity (Basmajian and De Luca 1985) may be negligible in this kind of experimental paradigm as demonstrated in Cannon and Zahalak (1982).

Reflexes could be another source of modeling error. Because of the low-pass-filter characteristics of nerve impulses and muscle force (Mannard and Stein 1973), a part of the stiffness components could be attributed to reflex despite the minor correlation between the EMG and angular position shown in the preceding section. The magnitude of the reflex contribution to total stiffness is controversial. Sinkjaer and Hayashi (1989) estimated that 30 to $>50 \%$ of wrist stiffness can be attributed to reflexes by abolishing the stretch reflex through ischemia. Similar values were obtained from the results of Bennett (1994) for the elbow. On the other hand, Soechting, Dufresne, and Lacquaniti (1981) and Lacquaniti, Licata, and Soechting (1982) argued that the EMG attributed to reflex response is low (5-10\%). Lacquaniti et al. (1982) suggested that reflex contributes mainly to viscosity rather than stiffness. Furthermore, De Serres and Milner (1991) mentioned that they could find no evidence to suggest that reflex responses contribute significantly to joint stiffness. Different estimations of the influence of the reflex response on stiffness may be attributed to the perturbation properties.

We may need to include the nonlinearities and reflex effects if musculo-skeletal dynamics are to be modeled more accurately. However, the compact model introduced in this study is sufficient to characterize the major factors relating joint stiffness to muscle activation. This simple model is advantageous because we can use surface EMG signals to predict arm stiffness instead of using direct measurements (Osu et al. 1997).

\section{Regulation of biarticular muscle activities}

The role and regulation mechanism of biarticular muscles have been one of the major topics in human arm-movement studies, especially because the activation of biarticular muscles has been revealed to play an important role in the formation of hand-stiffness stability (Hogan 1985; Mussa-Ivaldi et al. 1985).

From the viewpoint of muscle kinematics, biarticular muscles should be effective only in the force directions where the torque directions at both the shoulder and elbow are same because each biarticular muscle rotates the both joints in the same direction. If this rule is adopted in the CNS, biarticular muscles should not be activated in force directions 5, 6, 7, 13, 14 , and 15 where the shoulder and elbow rotations are opposite. However, the biarticular flexor often was activated in force directions 13,14, and 15, and the biarticular extensor often was activated in force directions 5, 6, and 7, except for subject $B$. In addition, the biarticular muscles' preferred force directions were biased to those of the elbow monoarticular muscles rather than those of shoulder monoarticular muscles. The higher correlation between bi- and monoarticular effective muscle stiffness in the elbow than in the shoulder indicates that biarticular muscle activations covary more with the elbow monoarticular muscles than with the shoulder monoarticular muscles (Fig. 6). In the shoulder joint, biarticular muscles did not always activate simultaneously with monoarticular muscles but sometimes were inactivated when monoarticular muscles were activated. These observations suggest that the CNS activates biarticular muscles mainly to control the elbow joint 
rather than the shoulder joint. As a result of the elbow-oriented regulation of biarticular muscles, the shoulder antagonists should cancel the shoulder torque generated by biarticular muscles. This cancellation of shoulder torque typically was observed for all subjects in force direction 13 (see Fig. 5). In this force direction, the shoulder monoarticular extensor was activated to cancel the torque generated by the biarticular flexor because no shoulder torque was required.

The biarticular muscles' preference of elbow joint to shoulder joint could be ascribed to their different moment arm for each joint. According to Meek et al. (1990), the effective moment arm of the biceps for the elbow flexion is larger than that for the shoulder flexion. This means that the biceps can rotate the elbow joint more effectively than the shoulder joint. This relation regarding the size of the effective moment arm is consistent with the parameters estimated here in tasks without cocontraction (parameters $a_{5}$ and $a_{7}$ ). The biased moment arms of biarticular muscles resulted in the smaller contribution of biarticular muscles to shoulder stiffness than to elbow stiffness observed in Fig. 6A. It may be reasonable to assume that biarticular muscles are intended for the joint for which they can more effectively generate torque (Buchanan et al. 1986). However, it is not quite certain whether this principle holds true for the triceps long head or for all biarticular muscles.

Another possibility to explain the preference of biarticular muscles is the synergy between biarticular muscles and elbow monoarticular muscles that has been reported in several studies. Wadman, Denier van der Gon, and Derksen (1980), Buchaman et al. (1986), and Flanders and Soechting (1990) reported a synergistic relationship between biceps and brachioradialis or brachialis. Buchaman et al. (1986) reported synergy between triceps long head and medial head for extensors. On the other hand, uncoupled activations of these muscles also have been reported. Jamison and Caldwell (1993) reported the reciprocal activation of biceps and brachioradialis during supination/pronation isometric torque productions. The reciprocal inhibitory projection has been found between the biceps and brachioradialis (Miyasaka et al. 1995; Naito et al. 1996). For the same pair of muscles to show both a synergistic relationship together with a reciprocal inhibitory relationship, some mechanism should exist to control alternative muscle regulations according to the torque requirements. The independent operation of each head of triceps also is suggested in several studies. Buchaman et al. reported different directionality in the three heads of triceps brachii. Flanders and Soechting (1990) reported quite different directional tuning between the long head and medial head of the triceps. The present study also found a difference in preferred direction between the long head and lateral head of triceps (see Fig. 5 and Table 2). Although the three head of the triceps brachii share the same tendon, their activations are not coupled and may be controlled separately by the CNS.

\section{Task-dependent regulation of muscle activation}

Task-dependent change in muscle regulation is another key topic in the field of human motor control. In isometric static force-regulation tasks, the ratio of single-joint stiffness to cross-joint stiffness changed with the force and/or cocontraction levels. However, the ratio was limited to a local range, i.e., single-joint stiffness was always higher than cross-joint stiffness (Gomi and Osu 1998). The limitation in the ratio of single- to cross-joint stiffness observed in isometric tasks results from the covaried activations of monoarticular and biarticular muscles. Cross-joint stiffness is composed of biarticular effective muscle stiffness, whereas single-joint stiffness is composed of both biarticular and monoarticular effective muscle stiffness. In the present isometric conditions, monoarticular muscles always were activated whenever biarticular muscles were activated (see Fig. 6). Note that monoarticular muscle activation was not always accompanied by biarticular muscle activation. From the constraints given by Eqs. 6 and 7, the parameter for each biarticular muscle's activation to cross-joint stiffness $\left(a_{9}, a_{10}\right)$ is smaller than the average of the parameters for its activation to single-joint stiffness $\left(a_{5}\right.$ and $a_{7}, a_{6}$ and $\left.a_{8}\right)$. The constraints mean that the biarticular effective muscle stiffness in cross-joint stiffness will not greatly exceed the biarticular effective muscle stiffness in single-joint stiffness unless the moment arm of a particular biarticular at the shoulder joint muscle is quite different from that at the elbow joint. Thus single-joint stiffness as a whole always exceeds cross-joint stiffness due to the constraints and the covaried activations in isometric conditions.

Contrary to the observations in isometric conditions, it has been reported (Karst and Hasan 1991) that during some movements, biarticular muscles were activated vigorously, whereas the monoarticular muscle were less activated. If it is possible to assume that the muscle activation is proportional to the muscle stiffness during movements, the elbow single-joint stiffness will decrease compared with cross-joint stiffness. In addition, direct observation of the decrease in elbow single-joint stiffness during a constrained movement has been reported (Gomi and Kawato 1995, 1996a). The regulation of muscle activation in static conditions seems to be quite different from that during movements.

Tax et al. (1989, 1990a,b) and van Groeningen and Erkelens (1994) showed that recruitment thresholds in biarticular muscles are higher in isometric conditions than during movement. Their observations are consistent with our prediction that in static tasks, the activation of monoarticular muscles is facilitated whereas during movement, the activation of biarticular muscles is facilitated.

The advantage of activating both mono- and biarticular muscles at the elbow-joint in static tasks may be ascribed to the increases in stability and/or controllability that are required in the force-regulation tasks. Additionally, the CNS may try to distribute effort among muscles to avoid fatiguing a particular muscle. A more detailed examination of the task-dependent difference in muscle activation pattern is required to reveal the principle on which the CNS regulates multiple muscle systems.

\section{A P P E N D I X}

\section{A. Normalization of EMG signals}

The EMG signals measured on different days were normalized and scaled by the following method. We measured the EMG signals during applications of a $15 \mathrm{~N}$ hand force at the position where the shoulder joint angle was $45^{\circ}$ and the elbow joint angle was $90^{\circ}$ on each experimental day for each subject (see Fig. A1). The directions of the hand force were $135^{\circ}$ (the direction opposite the elbow rotational center, direction 1 in Fig. A1), $315^{\circ}$ (the direction of the elbow rotational center, direction 2 ), $225^{\circ}$ (the direction parallel to the upper arm, direction 3 ), $45^{\circ}$ (the direction parallel to the upper arm, direction 


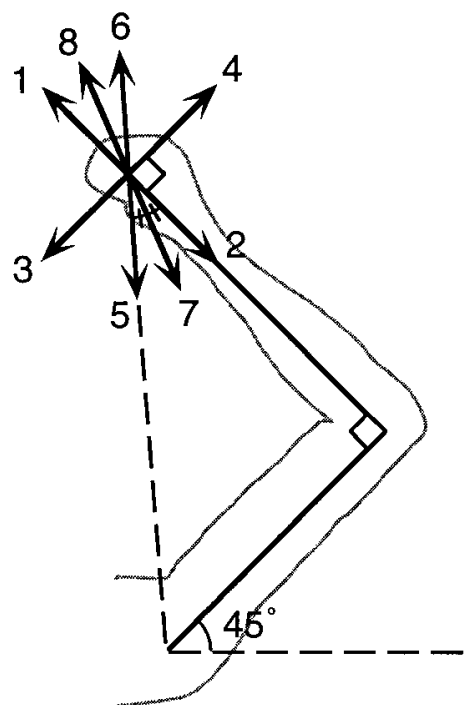

FIG. A1. Hand-force directions during reference EMG signal measurement for normalization (explanation in text).

4), the direction of the shoulder rotational center (direction 5), the direction opposite the shoulder rotational center (direction 6), the direction between the shoulder rotational center and elbow rotational center (direction 7), and the opposite direction between shoulder rotational center and elbow rotational center (direction 8). The EMG signals rectified and averaged for each force direction were called "reference EMG level." The reference EMG level on one experimental day was used as the "standard EMG level" and EMG signals of the other days were normalized to the standard EMG level by using the reference EMG level measured on the corresponding day. These EMG signals normalized to standard EMG level were then scaled for each muscle by the value of maximum voluntary contraction.

The correlation coefficients between the standard EMG level and the EMG level reconstructed from reference EMG level for each day were $0.984,0.969$, and 0.978 for subject $A, 0.987$ and 0.983 for subject $B, 0.988,0.969$, and 0.990 for subject $C$, and 0.991 and 0.994 for subject $D$.

\section{Estimation method}

Equation 1 can be derived from the following relationship among joint torque and muscle tension, muscle stiffness, and muscle length. Muscle tension $\mathbf{T}$ can be determined from muscle stiffness $\mathbf{S}$ and the stretch length of each muscle $\left(\mathbf{L}_{0}-\mathbf{L}\right)$ as follows.

$$
\begin{gathered}
\mathbf{T}=\mathbf{S} \cdot\left(\mathbf{L}_{0}-\mathbf{L}\right) \\
\mathbf{L}=\mathbf{L}(0)-\mathbf{L}(\mathbf{q})=\mathbf{A}^{T} \mathbf{q} \\
\mathbf{L}_{0}=\mathbf{l}_{\text {bias }}-\mathbf{l}_{\mathrm{eq}} \\
=\mathbf{l}_{\text {bias }}-\left(\mathbf{l}_{0}-\mathbf{l u}\right) \\
=\mathbf{l}_{\text {bias }}^{*}+\mathbf{l} \mathbf{u}
\end{gathered}
$$

Here, $\mathbf{L}(0)$ denotes the muscle length when the joint angle is $0, \mathbf{L}(\mathbf{q})$ denotes the current muscle length, and $\mathbf{q}$ denotes the current joint angle. $\mathbf{l}_{\mathrm{eq}}$ denotes the equilibrium length of a muscle (assumed to be a linear function of motor command $\mathbf{u})$. $\mathbf{l}_{0}$ is the equilibrium length when motor command $\mathbf{u}$ is zero, and $\mathbf{l}$ is a constant. $\mathbf{l}_{\text {bias }}^{*}\left(=\mathbf{l}_{\text {bias }}-\mathbf{l}_{0}\right)$ is a bias term that is set so that $\mathbf{L}_{0}-\mathbf{L}$ is not negative. Joint torque $\tau$ can be determined as

$$
\begin{aligned}
\tau & =\mathbf{A} \cdot \mathbf{T} \\
& =\mathbf{A} \cdot \mathbf{S} \cdot\left(\mathbf{l}_{\text {bias }}^{*}+\mathbf{l u}-\mathbf{A}^{T} \mathbf{q}\right)
\end{aligned}
$$

The following are detailed expressions of parameters $a_{\mathrm{i}}$ and $b_{\mathrm{i}}$ in Eq. 5.

$$
\begin{gathered}
a_{1}=d_{1}^{2} p_{1}, \ldots, a_{i}=d_{\mathrm{i}}^{2} p_{i}, \ldots, a_{6}=d_{6}^{2} p_{6} \\
a_{7}=d_{7}^{2} p_{5}, a_{8}=d_{8}^{2} p_{6}, a_{9}=d_{5} d_{7} p_{5}, a_{10}=d_{6} d_{8} p_{6} \\
b_{1}=d_{1}^{2} s_{01}+d_{2}^{2} s_{02}+d_{5}^{2} s_{05}+d_{6}^{2} s_{06} \\
b_{2}=d_{5} d_{7} s_{05}+d_{6} d_{8} s_{06} \\
b_{3}=d_{3}^{2} s_{03}+d_{4}{ }^{2} s_{04}+d_{7}{ }^{2} s_{05}+d_{8}{ }^{2} s_{06}
\end{gathered}
$$

We thank Drs. M. Kawato of ERATO and ATR, H. Imamizu of ERATO, K. Kawano of CREST, and M. Honda, K. Ishii, and Y. Tohkura of Nippon Telegraph and Telephone for continuing encouragement and N. Imamura, H. Nagaoka of KOBELCO and T. Yoshioka of CSK Corporation for technical support.

Address for reprint requests: R. Osu, Kawato Dynamic Brain Project, ERATO, JST, c/o ATR 2-2 Hikaridai, Seika-cho, Soraku-gun, Kyoto, 6190288, Japan.

Received 19 February 1998; accepted in final form 2 December 1998.

\section{REFERENCES}

Basmajian, J. V. And De LuCA, C. J. Muscles Alive (5th ed.). Baltimore: Williams and Wilkins, 1985.

BENNET, D. J. Stretch reflex responses in the human elbow joint during a voluntary movement. J. Physiol. (Lond.) 474: 339-351, 1994.

Buchanan, T. S., Almdale, D.P.J., Lewis, J. L., AND Rymer, W. Z. Characteristics of synergic relations during isometric contractions of human elbow muscles. J. Neurophysiol. 56: 1225-1241, 1986.

CANNON, S. C. AND ZAHALAK, G. I. The mechanical behavior of active human skeletal muscle in small oscillations. J. Biomech. 15: 111-121, 1982.

De Serres, S. J. And Milner, T. E. Wrist muscle activation patterns and stiffness associated with stable and unstable mechanical loads. Exp. Brain Res. 86: 451-458, 1991.

Dolan, J. M., Friedman, M. B., And Nagurka, M. L. Dynamic and loaded impedance components in the maintenance of human arm posture. IEEE Trans. Systems Man Cybern. 23: 698-709, 1993.

Flanders, M. And Soechting, J. F. Arm muscle activation for static forces in three-dimensional space. J. Neurophysiol. 64: 1818-1837, 1990.

Flash, T. AND Mussa-Ivaldi, F. Human arm stiffness characteristics during the maintenance of posture. Exp. Brain Res. 82: 315-326, 1990.

Gomi, H. And Kawato, M. Task Dependent Stiffness of Human Multi-Joint Arm During Point-to-Point Movement. Kanagawa, Japan: NTT Basic Res. Labs. Information Science Res. Lab. 1995. (Technical Report No. ISRL 95-4).

Gomi, H. And Kawato, M. Equilibrium-point control hypothesis examined by measured arm stiffness during multijoint movement. Science 272: 117-120, 1996 .

Gomi, H. AND Kawato, M. Mechanical impedance of human arm during multi-joint movement in horizontal plane (in Japanese). J. Soc. Instrument Control Eng. 32: 369-378, 1996b.

Gomi, H. AND Kawato, M. Human arm stiffness and equilibrium-point trajectory during multi-joint movement. Biol. Cybern. 76: 163-171, 1997.

Gomi, H. AND Osu, R. Task dependent viscoelasticity of human multijoint-arm and its spatial characteristics for interaction with environments. J. Neurosci. 18: 8965-8978, 1998.

Hogan, N. The mechanics of multi-joint posture and movement control. Biol. Cybern. 52: 315-331, 1985.

Jamison, J. C. And CALdwell, G. E. Muscle synergies and isometric torque production: influence of supination and pronation level on elbow flexion. J. Neurophysiol. 70: 947-960, 1993.

JOYCE, G. C. AND RACK, P.M.H. Isotonic shortening and lengthening movements of cat soleus muscle. J. Physiol. (Lond.) 204: 475-491, 1969.

Kanosue, K., Yoshida, M., Akazawa, K., And Fujin, K. The number of active motor units and their firing rates in voluntary contraction of human brachialis muscle. Jpn. J. Physiol. 29: 427-443, 1979.

KARST, G. M. AND HASAN, Z. Timing and magnitude of electromyographic activity for two-joint arm movements in different directions. J. Neurophysiol. 66: 1594-1604, 1991.

Kearney, R. E. AND Hunter, I. W. System identification of human triceps surae stretch reflex dynamics. Exp. Brain Res. 51: 117-127, 1983. 
Kearney, R. E. and Hunter, I. W. System identification of stretch reflex dynamics. Crit. Rev. Biomed. Eng. 18: 55-87, 1990.

Lacquaniti, F., Carrozzo, M., and Borghese, N. A. Time-varying mechanical behavior of multijointed arm in man. J. Neurophysiol. 69: 1443-1464, 1993.

Lacquaniti, F., Licata, F., And Soechting, J. F. The mechanical behavior of the human forearm in response to transient perturbations. Biol. Cybern. 44: $35-46,1982$.

Mannard, A. And Stein, R. B. Determination of the frequency response of isometric soleus muscle in the cat using random nerve stimulation. J. Physiol. (Lond.) 229: 275-296, 1973.

Matthews, P.B.C. And Stein, R. B. The sensitivity of muscle spindle afferents to small sinusoidal changes of length. J. Physiol. (Lond.) 200: 723-743, 1968.

McIntyre, J., Mussa-Ivaldi, F. A., And Bizzi, E. The control of stable postures in the multijoint arm. Exp. Brain Res. 110: 248-264, 1996.

Meek, S. G., Wood, J. E., And Jacobsen, S. C. Model-based, multi-muscle EMG control of upper-extremity prostheses. In: Multiple Muscle Systems: Biomechanics and Movement Organization, edited by J. M. Winters and S. L.-Y. Woo. New York: Springer-Verlag, 1990, p. 360-376.

Milner, T. E., Cloutier, C., Leger, A. B., AND Franklin, D. W. Inability to activate muscles maximally during cocontraction and the effect on joint stiffness. Exp. Brain Res. 107: 293-305, 1995.

Miyasaka, T., Sun, Y.-J., Naito, A., Morita, H., Shindo, M., Shimizu, Y., AND YANAGISAWA, N. Reciprocal inhibition between biceps brachii and brachioradialis in the human. In: 4th IBRO World Congress of Neuroscience, Oxford: Rapid Communications 1995, p. 334.

Mussa-Ivaldi, F. A., Hogan, N., AND Bizzi, E. Neural, mechanical, and geometric factors subserving arm posture in humans. J. Neurosci. 10: 2732-2743, 1985.

Naito, A., Shindo, M., Miyasaka, T., Sun, Y.-J., and Morita, H. Inhibitory projection from brachioradialis to biceps brachii motoneurons in human. Exp. Brain Res. 111: 483-486, 1996.

Osu, R., Gomi, H., Domen, K., Yoshioka, T., and Kawato, M. Decrease in joint stiffness during motor learning revealed from measured EMG signals. Soc. Neurosci. Abstr. 23: 202, 1997.
SinkJaer, T. AND Hayashi, R. Regulation of wrist stiffness by the stretch reflex. J. Biomech. 22: 1133-1140, 1989.

Soechting, J. F., Dufresne, J. R., And Lacquaniti, F. Time-varying properties of myotatic response in man during some simple motor tasks. J. Neurophysiol. 46: 1226-1243, 1981.

Stein, R. B., Hunter, I. W., Lafontaine, S. R., and Jones, L. A. Analysis of short-latency reflexes in human elbow flexor muscles. J. Neurophysiol. 73: 1900-1911, 1995.

Tax, A.A.M., Denier van der Gon, J. J., ANd ERkelens, C. J. Differences in coordination of elbow flexor muscles in force tasks and in movement tasks. Exp. Brain Res. 81: 567-572, 1990a.

TaX, A.A.M., Denier van der Gon, J. J., Gielen, C.C.A.M., And Kleyne, M. Differences in central control of $\mathrm{m}$. biceps brachii in movement tasks and force tasks. Exp. Brain Res. 79: 138-142, 1990b.

TaX, A.A.M., Denier van Der Gon, J. J., Gielen, C.C.A.M., and TemPel, C.C.M.v.D. Differences in the activation of m. biceps brachii in the control of slow isotonic movements and isometric contractions. Exp. Brain Res. 76: $55-63,1989$

Tsuji, T., Morasso, P. G., Goto, K., and Ito, K. Human hand impedance characteristics during maintained posture. Biol. Cybern. 72: 475-485, 1995.

Tsuji, T., Moritani, M., KaneKo, M., And Ito, K. An analysis of human hand impedance characteristics during isometric muscle contractions. (in Japanese). Trans. Soc. Instrument Control Eng. 32: 271-280, 1996.

van Groeningen, C.J.J.E. AND ERKelens, C. J. Task-dependent differences between mono- and bi-articular heads of the triceps brachii muscle. Exp. Brain Res. 100: 345-352, 1994.

van Zuylen, E. J., Gielen, C.C.A.M., and Denier van Der Gon, J. J. Coordination and inhomogeneous activation of human arm muscles during isometric torques. J. Neurophysiol. 60: 1523-1548, 1988.

Wadman, W. J., Denier van der Gon, J. J., And Derksen, R.J.A. Muscle activation patterns for fast goal-directed arm movements. J. Hum. Move. Stud. 6: 19-37, 1980.

WINTERS, J. M. Hill-based muscle models: a systems engineering perspective. In: Multiple Muscle Systems, edited by J. M. Winters and S. L.-Y. Woo. New York: Springer-Verlag, 1990, p. 69-93. 